\title{
Thermal instability in a ferrimagnetic resonator strongly coupled to a loop-gap microwave cavity
}

\author{
Cijy Mathai, ${ }^{1}$ Oleg Shtempluck, ${ }^{1}$ and Eyal Buks ${ }^{1}$ \\ ${ }^{1}$ Andrew and Erna Viterbi Department of Electrical Engineering, Technion, Haifa 32000 Israel
}

(Dated: August 9, 2021)

\begin{abstract}
We study nonlinear response of a ferrimagnetic sphere resonator (FSR) strongly coupled to a microwave loop gap resonator (LGR). The measured response in the regime of weak nonlinearity allows the extraction of the FSR Kerr coefficient and its cubic damping rate. We find that there is a certain range of driving parameters in which the system exhibits instability. In that range, selfsustained modulation of the reflected power off the system is generated. The instability is attributed to absorption-induced heating of the FSR above its Curie temperature.
\end{abstract}

\section{INTRODUCTION}

Ferromagnetic and ferrimagnetic resonators [1] 3] are widely employed in a variety of microwave (MW) devices, including narrow band oscillators [4], filters [5], and parametric amplifiers [6]. These resonators exhibit a variety of intriguing physical effects [7], including BoseEinstein condensation [8] and magneto-optical coupling 9 -12]. Here we study a strongly coupled hybrid system composed of a loop gap resonator (LGR) integrated with a ferrimagnetic sphere resonator (FSR) made of yttrium iron garnet (YIG) 13, 14]. We focus on the regime of nonlinear response. In section III below we explore the effect on nonlinear damping in the region of relatively weak microwave driving. An instability, which is observed with a much stronger driving, is reported in section IV below, and a theoretical model, which attributes the instability to a driving-induced heating, is presented.

Many nonlinear dynamical effects have been observed before in FSRs, including auto-oscillations [15, 16], optical cooling [17], frequency mixing [18, 19] and bistability 20 24]. The Suhl instability (of both first and second orders) has been observed with transverse microwave driving, whereas parallel pumping instability has been observed with longitudinal driving 25]. Applications of nonlinearity for quantum data processing have been explored in [26 33].

Heating a YIG sphere from room temperature to $400 \mathrm{~K}$ by microwave driving having power of $450 \mathrm{~mW}$ has been reported in 34]. At a Curie temperature given by $T_{\mathrm{c}}=$ $560 \mathrm{~K}$, YIG undergoes a phase transition between an ordered ferrimagnetic state (FS) and a disordered paramagnetic state (PS). Thermal instability was observed in a cavity magneto-mechanical system [35]. Microwave oscillations induced by injecting spin-polarized current [36] into a magnetic-multilayer structure have been reported in [37]. Self-excited oscillations induced by ohmic heating in a $\mathrm{Y}_{3} \mathrm{Fe}_{5} \mathrm{O}_{12} / \mathrm{Pt}$ bilayer nanowire have been investigated in 38. Imaging of heating induced by the spin Peltier effect has been demonstrated in [39].

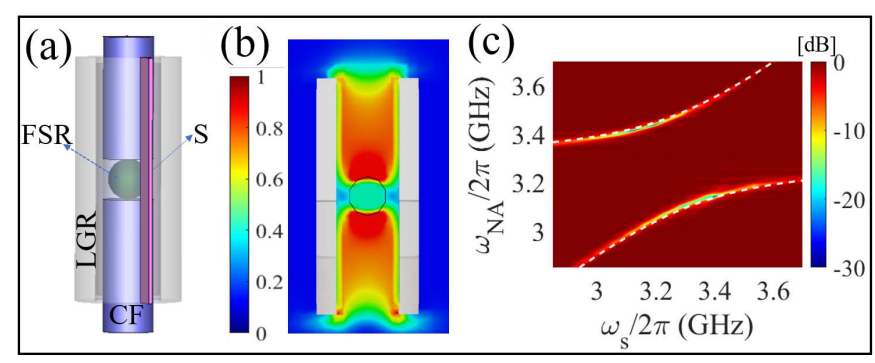

FIG. 1: FSR-LGR coupling: (a) A sketch of the FSR made of YIG having radius of $R_{\mathrm{S}}=1 \mathrm{~mm}$ that is integrated inside the aluminum cylindrical LGR having gap width of $0.3 \mathrm{~mm}$. The sphere is held by ceramic ferrules (CFs). A sapphire wafer (labeled as $\mathrm{S}$ ) is inserted into the gap to increase the capacitance. (b) The numerically calculated magnetic field energy density distribution (normalized with respect to the maximum value) corresponding to driving at the resonance frequency $\omega_{\mathrm{e}} /(2 \pi)=3.3 \mathrm{GHz}$. (c) A VNA reflectivity $\left|S_{11}\right|^{2}$ measurement as a function of magnon frequency $\omega_{s}$ (proportional to the externally applied magnetic field). The coupling coefficient $g_{\text {eff }}$ is extracted from the theoretical fit (white dashed lines) following Eq. (2).

\section{LOOP GAP RESONATOR}

With relatively low input power, the main mechanisms responsible for FSR nonlinear response are magnetic anisotropy [40] and exchange interaction [13]. Consider a MW cavity mode having angular frequency $\omega_{\mathrm{e}}$ and an integrated FSR having radius $R_{\mathrm{S}}$. It is assumed that the applied static magnetic field $\mathbf{H}_{\mathrm{s}}$ is parallel to the easy axis. In the Holstein-Primakoff approximation [41] (which assumes that magnetization is nearly saturated), the Hamiltonian of the system $\mathcal{H}_{\mathrm{D}}$ is expressed as 21, 42

$$
\begin{aligned}
\hbar^{-1} \mathcal{H}_{\mathrm{D}} & =\omega_{\mathrm{e}} N_{\mathrm{e}}+\omega_{\mathrm{s}} N_{\mathrm{s}}+K_{\mathrm{M}} N_{\mathrm{s}}^{2} \\
& +g_{\mathrm{eff}}\left(A_{\mathrm{e}}^{\dagger} A_{\mathrm{s}}+A_{\mathrm{e}} A_{\mathrm{s}}^{\dagger}\right),
\end{aligned}
$$

where $N_{\mathrm{e}}=A_{\mathrm{e}}^{\dagger} A_{\mathrm{e}}\left(N_{\mathrm{s}}=A_{\mathrm{s}}^{\dagger} A_{\mathrm{s}}\right)$ is a cavity mode (FSR Kittel mode) number operator, $\omega_{\mathrm{s}}=\gamma_{\mathrm{g}} H_{\mathrm{s}}$ is the Kittel mode angular frequency, $\gamma_{\mathrm{g}} / 2 \pi=27.98 \mathrm{GHz} \mathrm{T}^{-1}$ is 
the gyromagnetic ratio, $K_{\mathrm{M}}=\hbar \gamma_{\mathrm{g}}^{2} K_{\mathrm{c} 1} /\left(V_{\mathrm{s}} M_{\mathrm{s}}^{2}\right)$ is the anisotropy-induced Kerr frequency, $K_{\mathrm{c} 1}$ is the first-order anisotropy constant, $V_{\mathrm{s}}=4 \pi R_{\mathrm{s}}^{3} / 3$ is the volume of the sphere, $M_{\mathrm{S}}$ is the saturation magnetization, and $g_{\text {eff }}$ is the cavity-FSR coupling coefficient. For YIG at room temperature, $M_{\mathrm{s}}=140 \mathrm{kA} / \mathrm{m}$ and $K_{\mathrm{c} 1}=-610 \mathrm{~J} / \mathrm{m}^{3}$, hence $K_{\mathrm{M}}=-2.4 \times 10^{-8} \mathrm{~Hz} \times\left(R_{\mathrm{s}} /(100 \mu \mathrm{m})\right)^{-3}$.

In the linear regime, where the Kerr nonlinearity can be disregarded, the Hamiltonian $\mathcal{H}_{\mathrm{D}}$ (1) can be diagonalized. The angular frequencies $\omega_{ \pm}$of the two hybrid photon-magnon eigen modes are given by [43]

$$
\omega_{ \pm}=\frac{\omega_{\mathrm{e}}+\omega_{\mathrm{s}}}{2} \pm \sqrt{\left(\frac{\omega_{\mathrm{e}}-\omega_{\mathrm{s}}}{2}\right)^{2}+g_{\mathrm{eff}}^{2}} .
$$

Both angular frequencies $\omega_{ \pm}$are positive provided that $g_{\text {eff }}<\sqrt{\omega_{\mathrm{s}} \omega_{\mathrm{e}}}$. Note that the super-radiance Dicke instability occurs in the ultra-strong coupling region where $g_{\text {eff }}>\sqrt{\omega_{\mathrm{s}} \omega_{\mathrm{e}}}$ [44]. In the rotating wave approximation (RWA) the Kerr coefficients $K_{ \pm}$of the hybrid modes having angular frequencies $\omega_{ \pm}$are given by Eqs. (A9) and (A10) of appendix A [see Eq. A8)].

In the current experiment, we explore the response for a wide range of the MW input powers $P_{\mathrm{p}}$. We find that the response is well described by the Hamiltonian $\mathcal{H}_{\mathrm{D}}$ provided that $P_{\mathrm{p}}$ is sufficiently small. However, with sufficiently high $P_{\mathrm{p}}$, the FSR temperature $T$ may exceed the Curie temperature $T_{\mathrm{c}}$ due to MW absorption-induced heating. We study the response of the FSR-LGR system to an injected monochromatic pump tone having a frequency close to resonance. The off reflected power is measured using a spectrum analyzer (SA). We find that there is a certain zone in the pump frequency - pump amplitude plane, in which the resonator exhibits limit-cycle (LC) response resulting in self-sustained modulation of the reflected power. The observed LC is attributed to thermal instability (TI) 45].

A MW cavity made of an LGR allows achieving a relatively large coupling coefficient $g_{\text {eff }}$ [46, 47]. The MW LGR schematically shown in Fig. 1(a), is made of a hollow concentric aluminium tube having an inner and outer radii of $R_{\mathrm{LGR}}=1.7 \mathrm{~mm}$ and $3 \mathrm{~mm}$, respectively, and a height of $H_{\mathrm{LGR}}=12 \mathrm{~mm}$. A sapphire strip of $260 \mu \mathrm{m}$ thickness has been inserted into the gap in order to increase its capacitance, which in turn reduces the frequency $f_{\mathrm{e}}$ of the LGR fundamental mode $\left[f_{\mathrm{e}}=\omega_{\mathrm{e}} /(2 \pi)=3.3 \mathrm{GHz}\right.$ with sapphire] [48]. An FSR made of YIG having radius of $R_{\mathrm{S}}=1 \mathrm{~mm}$ is held by two ferrules inside the LGR. The static magnetic field $\mathbf{H}_{\mathrm{s}}$ is applied perpendicularly to the LGR axis. The LGR-FSR coupled system has been encapsulated in a metallic rectangular shield made of aluminum. The cavity is weakly coupled to a loop antenna (LA).

The numerically calculated magnetic energy density distribution corresponding to the LGR fundamental mode is shown in Fig. 1(b). The calculated density is homogeneous $(\simeq 95 \%)$ over the FSR volume, and it is well confined inside the LGR inner volume. Note that for our device, the LGR inner volume, which is given by $\pi R_{\mathrm{LGR}}^{2} H_{\mathrm{LGR}}$, is 4 orders of magnitude smaller than the volume $\lambda_{\mathrm{e}}^{3}$, where $\lambda_{\mathrm{e}}=c / f_{\mathrm{e}}$ is the free space wavelength corresponding to the LGR frequency $f_{\mathrm{e}}$, and $c$ is the speed of light in vacuum. Consequently, the coupling coefficient $g_{\text {eff }}$ can be made much larger than typical values obtained with the commonly employed rectangular cavities [28], for which the mode volume commonly has the same order of magnitude as $\lambda_{\mathrm{e}}^{3}$.

Based on Eq. (2) of Ref. [28], together with the evaluated energy density shown in Fig. 1(b), the calculated value of the coupling coefficient is found to be $g_{\text {eff }}=176 \mathrm{MHz}$ for the LGR fundamental mode of frequency $f_{\mathrm{e}}=3.3 \mathrm{GHz}$. Alternatively, $g_{\text {eff }}$ can be extracted from measurements of MW reflection coefficient $\left|S_{11}\right|^{2}$ as a function of the Kittel mode frequency $\omega_{\mathrm{s}} /(2 \pi)$ and driving frequency $\omega_{\mathrm{NA}} /(2 \pi)$. Fitting $\left|S_{11}\right|^{2}$, which is measured at temperature of $3 \mathrm{~K}$ using a vector network analyzer (VNA), with Eq. (2) [see Fig. 1(c)] yields the value $g_{\text {eff }}=200 \mathrm{MHz}$, which is pretty much close to the value obtained from simulation. Note that $g_{\text {eff }}$ is only one order of magnitude smaller than the threshold value corresponding to the super-radiance Dicke instability [44].

\section{KERR COEFFICIENT AND NONLINEAR DAMPING}

Cavity driving having amplitude $\Omega_{\mathrm{p}}$ and angular frequency $\omega_{\mathrm{p}}$ is taken into account by adding a term given by $\hbar \Omega_{\mathrm{p}}\left(A_{\mathrm{e}}^{\dagger} e^{-i \omega_{\mathrm{p}} t}+A_{\mathrm{e}} e^{-i \omega_{\mathrm{p}} t}\right)$ to the Hamiltonian $\mathcal{H}_{\mathrm{D}}$ (11). Steady state solution of the driven system was calculated in Ref. [40] for the case where damping is taken into account to first order only. For that case the solution is found by solving a cubic equation for the FSR dimensionless energy $E_{\mathrm{s}}=\left\langle N_{\mathrm{s}}\right\rangle$ [given by Eq. (36) of [40]]. We find, however, that the calculated steady state yields only a moderate agreement with experimental data. Better agreement can be obtained by taking into account nonlinear damping to cubic order [49]. In this approach the cubic equation for $E_{\mathrm{s}}$ becomes

$$
\left(\delta_{\mathrm{s}}^{\prime 2}+\gamma_{\mathrm{s}}^{\prime 2}\right) E_{\mathrm{s}}=\eta\left|\Omega_{\mathrm{p}}\right|^{2},
$$

where $\delta_{\mathrm{s}}^{\prime}=\delta_{\mathrm{s}}-\eta \delta_{\mathrm{e}}+2 K_{\mathrm{M}} E_{\mathrm{s}}, \quad \delta_{\mathrm{s}}=\omega_{\mathrm{s}}-\omega_{\mathrm{p}}$ and $\delta_{\mathrm{e}}=\omega_{\mathrm{e}}-\omega_{\mathrm{p}}$ are driving detuning angular frequencies, $\eta=g_{\mathrm{eff}}^{2} /\left(\delta_{\mathrm{e}}^{2}+\gamma_{\mathrm{e}}^{2}\right), \gamma_{\mathrm{e}}=\gamma_{1 \mathrm{e}}+\gamma_{2 \mathrm{e}}$ with $\gamma_{1 \mathrm{e}}\left(\gamma_{2 \mathrm{e}}\right)$ being the external (intrinsic) cavity damping rate, $\gamma_{\mathrm{s}}^{\prime}=$ $\gamma_{\mathrm{s}}+\eta \gamma_{\mathrm{e}}+\gamma_{3 \mathrm{~s}} E_{\mathrm{s}}, \gamma_{\mathrm{s}}$ is the FSR linear damping rate and $\gamma_{3 \mathrm{~s}}$ is the FSR cubic nonlinear damping coefficient. Note that $\left|\Omega_{\mathrm{p}}\right|^{2}$ is proportional to the driving power $P_{\mathrm{p}}$ injected into the LA. Note also that when nonlinear damping is disregarded (i.e. when $\gamma_{3 \mathrm{~s}}=0$ ) Eq. (3) becomes identical to Eq. (36) of [40].

VNA measurements of the reflection coefficient $\left|S_{11}\right|^{2}$ for three different values of $P_{\mathrm{p}}$ are shown in Fig. 2(a-c). For the data presented in both Fig. 22 and Fig. 3, the radius of the FSR is $R_{\mathrm{S}}=0.1 \mathrm{~mm}$. The theoretical fit 


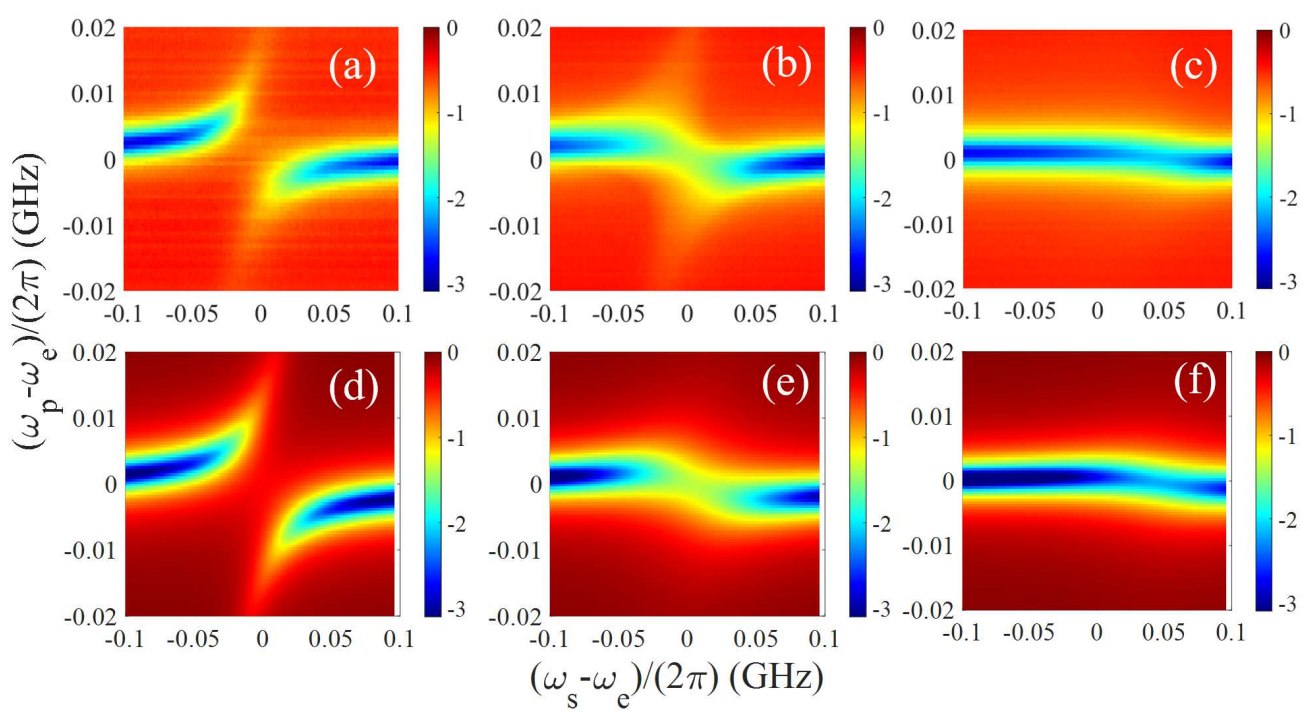

FIG. 2: Reflection coefficient $\left|S_{11}\right|^{2}$ in dB units for three values of MW input power $P_{\mathrm{p}}$. Panels (a), (b), and (c) present the experimental data corresponding to $\mathrm{MW}$ input powers $P_{\mathrm{p}}$ of $-20 \mathrm{dBm},-5 \mathrm{dBm}$, and $+10 \mathrm{dBm}$, respectively. The second row [panels (c), (d), and (e)] shows the corresponding theoretical fits that are obtained from Eq. (3). The theoretical fit parameters are $\gamma_{2 \mathrm{e}}=1.5 \mathrm{MHz}, \gamma_{\mathrm{e}}=4 \mathrm{MHz}, \gamma_{\mathrm{s}}=1 \mathrm{MHz}, K_{\mathrm{M}}=6.325 \mathrm{nHz}, \delta_{\mathrm{e}}=35 \mathrm{MHz}$, and $\gamma_{3 \mathrm{~s}}=0.001 \mathrm{nHz}$. To obtain a proper fit, $N_{\mathrm{s}}$ and $g_{\mathrm{eff}}$ are taken as variable values varying as a function of $P_{\mathrm{p}}$. For $P_{\mathrm{p}}=-20 \mathrm{dBm},-5 \mathrm{dBm}$, and $10 \mathrm{dBm}, N_{\mathrm{s}}$ values are taken as $1 \times 10^{19} \mathrm{~m}^{-3}, 5 \times 10^{19} \mathrm{~m}^{-3}$ and $8 \times 10^{19} \mathrm{~m}^{-3}$, and $g_{\text {eff }}$ values are taken as $14 \mathrm{MHz}, 14 \mathrm{MHz}$ and $12 \mathrm{MHz}$, respectively.

shown in Fig. 2(d-f) is based on the cubic equation (3), which allows the calculation of the dimensionless energy $E_{\mathrm{s}}$, and on Eq. (3) of Ref. [28], which evaluates the reflection coefficient $\left|S_{11}\right|^{2}$ as a function of $E_{\mathrm{s}}$. The values of parameters assumed for the calculations are listed in the caption of Fig. 22 Note the driving-induced blue shift observed in the magnetic resonance frequency [see Fig. 2(a-c)]. This shift cannot be accurately reproduced theoretically when nonlinear damping is disregarded.

\section{THERMAL INSTABILITY}

Further insight can be gained by measuring the spectral density $I_{\mathrm{SA}}$ of the signal reflected off the LA using a SA (see Fig. 3). We find that for $P_{\mathrm{p}}>P_{\mathrm{c}}=42.5 \mathrm{dBm}$, and for sufficiently small detuning from resonance, the measured spectral density $I_{\mathrm{SA}}$ contains equally-spaced side-bands (SB) on both sides of the driving frequency $f_{\mathrm{p}}=\omega_{\mathrm{p}} /(2 \pi)$ [see Fig. 3(a)]. We measure the SB spacing frequency $\omega_{\mathrm{SM}} /(2 \pi)$ as a function of the driving frequency $f_{\mathrm{p}}$ and driving power $P_{\mathrm{p}}$ [see Fig. 3(c)].

The observed equally spaced SBs are attributed to a thermal instability mechanism that is discussed in Ref. [45]. The phase transition occurring at the Curie temperature $T_{\mathrm{c}}$ between the FS and the PS gives rise to a sharp change in the resonance modes of the hybrid cavity-FSR system. Consider the case where the frequency of the externally applied driving is tuned very close to the frequency of one the hybrid system modes. With sufficiently high driving amplitude the temperature
$T$ of the FSR may exceeds the Curie temperature $T_{\mathrm{c}}$ due to driving-induced heating. For that case no steady state with $T<T_{\mathrm{c}}$ (i.e. FS) exists. The transition from the FS to the PS occurring at $T_{\mathrm{c}}$ is expected to give rise to a resonance frequency shift. Consequently the driving-induced heating is expected to abruptly drop down, since above $T_{\mathrm{c}}$ the frequency detuning between the continuous wave external driving and the resonance frequency becomes larger (in absolute value). Consider the case where the reduced heating gives rise to a temperature drop below $T<T_{\mathrm{c}}$. For this case, a steady state with $T>T_{\mathrm{c}}$ (i.e. PS) also becomes impossible. In the region where no steady state is possible, the temperature is expected to oscillate around $T_{\mathrm{c}}$. The frequency of temperature oscillation can be determined from the spacing between the measured SBs.

For the measurements presented in Fig. 3, the driving angular frequency $\omega_{\mathrm{p}}$ is tuned close to $\omega_{+}$. The analysis is greatly simplified by disregarding the other hybrid eigen mode having angular frequency $\omega_{-}$. This approximation is applicable in the strong coupling regime, for which the resonances having angular frequencies $\omega_{ \pm}$do not overlap [see Eq. (2)]. In this approach the FSR-cavity system is treated as a single mode having angular frequency $\omega_{+}=$ $2 \pi \times 3.32 \mathrm{GHz}$, and Kerr coefficient $K_{+}=K_{\mathrm{M}} \sin ^{4}\left(\theta_{\mathrm{g}} / 2\right)$ [see Eq. A99)]. The mode damping rate $\gamma_{+}=30 \mathrm{MHz}$ is expressed as $\gamma_{+}=\gamma_{1+}+\gamma_{2+}$, where $\gamma_{1+}$ is the coupling coefficient between the driven mode and the LA, and $\gamma_{2+}$ is the mode intrinsic damping rate (note that $\gamma_{1+}=\gamma_{2+}$ for critical coupling).

To account for the observed SB, we consider the ef- 

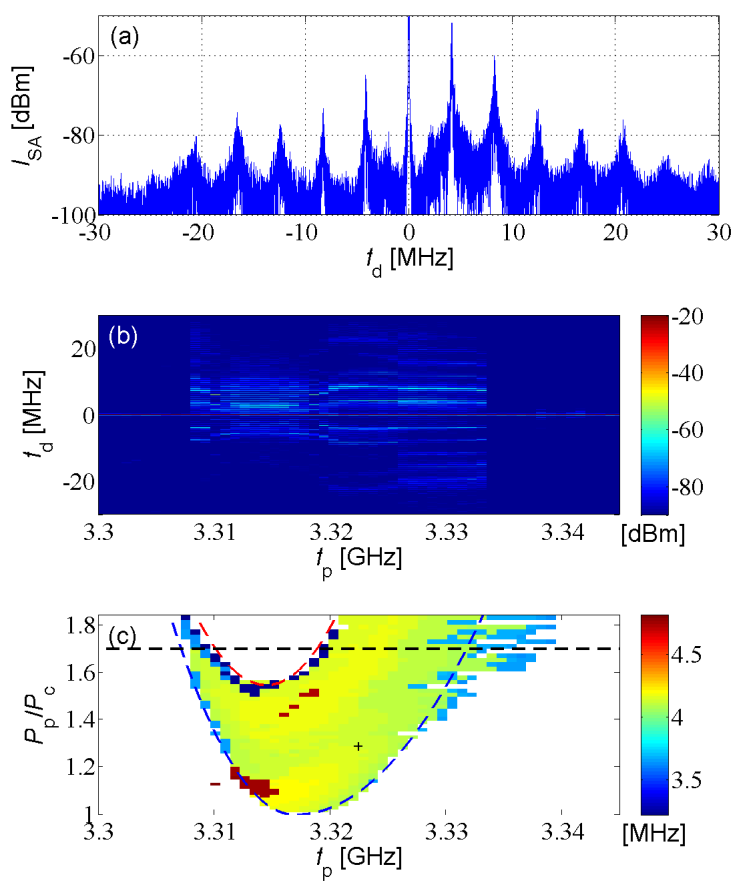

FIG. 3: Thermal instability. (a) Spectral density $I_{\mathrm{SA}}$ of the signal reflected off the LA, as a function of the detuning frequency $f_{\mathrm{d}}$, for the driving frequency $f_{\mathrm{p}}=3.2224 \mathrm{GHz}$ and normalized driving power $P_{\mathrm{p}} / P_{\mathrm{c}}=1.288$ specified by the black cross overlaid in (c). (b) Spectral density $I_{\mathrm{SA}}$ in $\mathrm{dB}$ as a function of the driving frequency $f_{\mathrm{p}}$ and detuning frequency $f_{\mathrm{d}}$ for $P_{\mathrm{p}} / P_{\mathrm{c}}=1.7$ [indicated by the overlaid horizontal dashed line in (c)]. (c) The SB spacing frequency $\omega_{\mathrm{SM}} /(2 \pi)$ in $\mathrm{MHz}$ as a function of driving frequency $f_{\mathrm{p}}$ and normalized driving power $P_{\mathrm{p}} / P_{\mathrm{c}}$. The overlaid blue (red) dashed line represents the threshold condition $E_{\mathrm{F}}=E_{\mathrm{cF}}\left(E_{\mathrm{P}}=\right.$ $\left.E_{\mathrm{cP}}\right)$. The following values are assumed for the calculations $\omega_{+\mathrm{F}} / 2 \pi=3.317 \mathrm{GHz}, \omega_{+\mathrm{P}} / 2 \pi=3.314 \mathrm{GHz}, \gamma_{+\mathrm{F}}=1.3 \times \gamma_{+\mathrm{P}}$, $\sigma_{\mathrm{F}} / w_{\mathrm{TF}}=2.6 \times \sigma_{\mathrm{P}} / w_{\mathrm{TP}},\left(K_{+\mathrm{F}} / \gamma_{+\mathrm{F}}\right)\left(w_{\mathrm{TF}} / \sigma_{\mathrm{F}}\right)=0.5$ and $K_{+\mathrm{P}}=0$.

fect of driving-induced heating on the FSR magnetic ordering. The externally applied driving gives rise to a heating power $Q$ given by $Q=2 \hbar \omega_{+} \gamma_{2+}|B|^{2}$, where $B$ is the complex amplitude of the driven mode (note that nonlinear damping is disregarded here). It is assumed that the FSR temperature $T$ is uniform, and that the cooling power due to the coupling between the FSR and its environment at a base temperature of $T_{0}$ is given by $H\left(T-T_{0}\right)$, where $H$ is the heat transfer coefficient. The thermal heat capacity of the FSR is denoted by $C$. It is assumed that all the parameters characterizing the mode abruptly change at a critical temperature given by $T_{\mathrm{c}}$. In the adiabatic (diabatic) region, the mode linear damping rate $\gamma_{+}$is much smaller (larger) than the thermal decay rate $H / C$.

In dimensionless form, system's time evolution is gov-
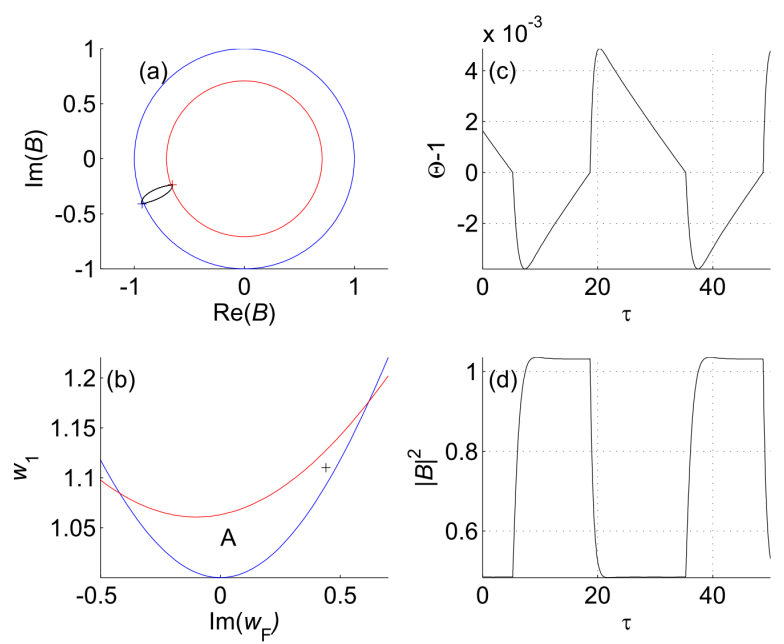

FIG. 4: Limit cycle. (a) Numerical integration of the equations of motion (4) and (5) is performed with the following parameters $\operatorname{Im}\left(w_{\mathrm{F}}-w_{\mathrm{P}}\right)=-0.1, \operatorname{Re}\left(w_{\mathrm{F}}\right)=-1, \operatorname{Re}\left(w_{\mathrm{P}}\right)=$ $-1.5, \sigma_{\mathrm{F}}=0.01, \sigma_{\mathrm{P}}=0.02$, and $w_{\mathrm{TF}}=w_{\mathrm{TP}}=0.01$. The values of driving detuning frequency $\operatorname{Im}\left(w_{\mathrm{F}}\right)$ and driving amplitude $w_{1}=w_{1 \mathrm{~F}}=w_{1 \mathrm{P}}$ are indicated by the black cross in (b). The LC is shown in (a) as a closed curve in the complex $B$ plane, in (c) as a periodic function of $\Theta-1$ vs. the normalized time $\tau$, and in (d) as a periodic function $|B|^{2}$ vs. $\tau$. The plane of driving frequency and driving amplitude is shown in (b). No steady state solution exists in the region between the blue and red curves (labeled as A).

erned by [45]

$$
\begin{aligned}
& \dot{B}=w B-w_{1}, \\
& \dot{\Theta}=\sigma|B|^{2}-w_{\mathrm{T}} \Theta .
\end{aligned}
$$

Overdot denotes a derivative with respect to a dimensionless time $\tau$, which is related to the time $t$ by $\tau=\gamma_{0} t$, where $\gamma_{0}$ is a constant rate. The dimensionless complex frequency $w$ is given by $w=$ $\left(i\left(\omega_{\mathrm{p}}-\omega_{+}-K_{+}|B|^{2}\right)-\gamma_{+}\right) / \gamma_{0}$, the dimensionless driving amplitude $w_{1}$ is given by $w_{1}=i \gamma_{0}^{-1} \sqrt{2 \gamma_{1+}} \Omega_{\mathrm{p}}$, the dimensionless temperature $\Theta$ is given by $\Theta=$ $\left(T-T_{0}\right) /\left(T_{\mathrm{c}}-T_{0}\right)$, the dimensionless heating coefficient $\sigma$ is given by $\sigma=2 \hbar \omega_{+} \gamma_{2+} \gamma_{0}^{-1} C^{-1}\left(T_{\mathrm{c}}-T_{0}\right)^{-1}$, and the dimensionless thermal rate $w_{\mathrm{T}}$ is given by $w_{\mathrm{T}}=$ $(H / C) / \gamma_{0}$.

The normalized parameters $w, w_{1}, \sigma$ and $w_{\mathrm{T}}$ are assumed to have a step function dependence on the temperature. Below (above ) the critical temperature $T_{\mathrm{c}}$, i.e. for $\Theta<1(\Theta>1)$, they take the values $w_{\mathrm{F}}, w_{1 \mathrm{~F}}, \sigma_{\mathrm{F}}$ and $w_{\mathrm{TF}}\left(w_{\mathrm{P}}, w_{1 \mathrm{P}}, \sigma_{\mathrm{P}}\right.$ and $\left.w_{\mathrm{TP}}\right)$, respectively. A steady state (i.e. time independent) solution below (above) the critical temperature $T_{\mathrm{c}}$, i.e. in the region $\Theta<1(\Theta>1)$, is possible provided that $E_{\mathrm{F}}<E_{\mathrm{cF}}\left(E_{\mathrm{P}}>E_{\mathrm{cP}}\right)$, where $E_{\mathrm{F}}=\left|w_{1 \mathrm{~F}} / w_{\mathrm{F}}\right|^{2}$ and $E_{\mathrm{CF}}=w_{\mathrm{TF}} / \sigma_{\mathrm{F}}\left(E_{\mathrm{P}}=\left|w_{1 \mathrm{P}} / w_{\mathrm{P}}\right|^{2}\right.$ and $\left.E_{\mathrm{cP}}=w_{\mathrm{TP}} / \sigma_{\mathrm{P}}\right)$ [see Eqs. (4) and (5) and Fig. 4(b)]. Note that both $E_{\mathrm{F}}$ and $E_{\mathrm{P}}$ represent steady state values 
of Eq. (41) for $|B|^{2}$, whereas both $E_{\mathrm{cF}}$ and $E_{\mathrm{cP}}$ represent values of $|B|^{2}$, for which $\Theta=1$ is a steady state value of Eq. (5).

Heat can be removed from the FSR by radiation, exchange with the surrounding air, and exchange with the supporting ferrules, which hold the FSR inside the LGR. The contributions to the total heat transfer coefficient $H$ due to radiation, air and the ferrules are denoted by $h_{\mathrm{rad}} S_{\mathrm{s}}, h_{\mathrm{air}} S_{\mathrm{s}}$ and $H_{\mathrm{fer}}$, respectively, where $S_{\mathrm{s}}=4 \pi R_{\mathrm{s}}^{2}$ is the FSR surface area. The coefficient $h_{\mathrm{rad}}$ is roughly given by $h_{\mathrm{rad}} \simeq \alpha_{\mathrm{YIG}} \sigma_{\mathrm{SB}}\left(T_{\mathrm{c}}^{4}-T_{0}^{4}\right) /\left(T_{\mathrm{c}}-T_{0}\right)$, where $\alpha_{\text {YIG }}$ is the averaged FSR absorption coefficient in the spectral band corresponding to room temperature $T_{0} \simeq 300 \mathrm{~K}$ radiation (wavelength $\lambda \simeq 10 \mu \mathrm{m}$ ), $\sigma_{\mathrm{SB}}=\pi^{2} k_{\mathrm{B}}^{4} /\left(60 \hbar^{3} c^{2}\right)$ is the Stefan-Boltzmann constant, $k_{\mathrm{B}}$ is the Boltzmann's constant, $\hbar$ is Plank's constant, and $T_{\mathrm{c}}=560 \mathrm{~K}$ is the YIG Curie temperature. The absorption coefficient value $\alpha_{\mathrm{YIG}} \simeq 10^{-1}[50]$ yields $h_{\mathrm{rad}} \simeq$ $2 \mathrm{Wm}^{-2} \mathrm{~K}^{-1}$. For ambient temperature and pressure $h_{\text {air }} \simeq 15 \mathrm{Wm}^{-2} \mathrm{~K}^{-1}$, hence $\left(h_{\text {rad }}+h_{\text {air }}\right) S_{\mathrm{s}}\left(T_{\mathrm{c}}-T_{0}\right) \simeq$ $0.6 \mathrm{~mW}$ for a FSR having radius $R_{\mathrm{s}}=0.1 \mathrm{~mm}$. In the region where $\mathrm{SB}$ are observed the induced heating power applied to the FSR is about 3 orders of magnitudes larger, hence $H \simeq H_{\text {fer }}$, i.e. both radiation and air have negligibly small contributions, and thus heat is mainly removed by the ferrules.

The thermal heat capacity of a FSR having radius $R_{\mathrm{s}}=0.1 \mathrm{~mm}$ and volume $V_{\mathrm{s}}=4 \pi R_{\mathrm{s}}^{3} / 3$ is given by $C=2.9 \times 10^{6} \mathrm{JK}^{-1} \mathrm{~m}^{-3} \times V_{\mathrm{s}}=1.2 \times 10^{-5} \mathrm{JK}^{-1} 51$, hence the thermal decay rate is roughly given by $H / C \simeq$ $320 \mathrm{~Hz} \times\left(Q_{\mathrm{c}} / \mathrm{W}\right)\left(\left(T_{\mathrm{c}}-T_{0}\right) /(260 \mathrm{~K})\right)^{-1}$, where $Q_{\mathrm{c}}$ is the heating power applied to the FSR, for which the steady state temperature is $T_{\mathrm{c}}$. Hence for the current device $(H / C) / \gamma_{+} \simeq 10^{-5}$, and thus the diabatic approximation is applicable.

A typical limit cycle ( $\mathrm{LC}$ ) in the diabatic regime is shown in Fig. [4 The LC is calculated by numerically integrating the equations of motion (4) and (5). The blue (red) cross shown in Fig. 4(a) indicates the steady state value $w_{1} / w$ of $B$ corresponding to the FS (PS), i.e. for $\Theta<1(\Theta>1)$, and the blue (red) circle represents the relation $|B|^{2}=E_{\mathrm{cF}}\left(|B|^{2}=E_{\mathrm{CP}}\right)$. In the plane of driving frequency and driving amplitude, which is shown in Fig. $4(\mathrm{~b})$, the blue and red curves are derived from the relations $E_{\mathrm{F}}=E_{\mathrm{cF}}$ and $E_{\mathrm{P}}=E_{\mathrm{cP}}$, respectively. In the region labeled as $\mathrm{A}$, no steady state solution to Eqs. (4) and (5) exists. The LC period time $\tau_{\mathrm{LC}}$ can be calculated by integrating Eqs. (4) and (5) over a single period. In the diabatic limit, one finds that $\tau_{\mathrm{P}} \simeq\left|w_{\mathrm{P}}\right|^{-1}+\left|w_{\mathrm{F}}\right|^{-1}$. The measured value of $\mathrm{LC}$ frequency roughly agrees with this theoretical estimation.

\section{SUMMARY}

In summary, we demonstrate that relatively large coupling coefficient $g_{\text {eff }}$ can be obtained by employing an
LGR having mode volume much smaller than $\lambda_{\mathrm{e}}^{3}$. The response of the system in the weak nonlinear regime allows the extraction of the Kerr coefficient $K_{\mathrm{M}}$ and the cubic nonlinear damping rate $\gamma_{3 s}$. An instability is revealed by driving the system with a relatively high input power. Above the instability threshold the response of the system to an externally applied monochromatic driving exhibits self-modulation. The instability, which is attributed to driving-induced heating, occurs in a region where the response has no steady state value. Further study will be devoted to developing sensors that exploit this instability for performance enhancement.

\section{ACKNOWLEDGMENTS}

This work was supported by the Israeli science foundation, the Israeli ministry of science, and by the Technion security research foundation.

\section{Appendix A: Rotating wave approximation}

The Hamiltonian (1) can be expressed as

$$
\hbar^{-1} \mathcal{H}_{\mathrm{D}}=\left(\begin{array}{ll}
A_{\mathrm{e}}^{\dagger} & A_{\mathrm{s}}^{\dagger}
\end{array}\right) M\left(\begin{array}{c}
A_{\mathrm{e}} \\
A_{\mathrm{s}}
\end{array}\right)+K_{\mathrm{M}} N_{\mathrm{s}}^{2},
$$

where the $2 \times 2$ matrix $M$ is given by

$$
M=\left(\begin{array}{cc}
\omega_{\mathrm{e}} & g_{\mathrm{eff}} \\
g_{\mathrm{eff}} & \omega_{\mathrm{s}}
\end{array}\right) .
$$

The eigenvalues $\omega_{ \pm}$of the matrix $M$ are given by $\omega_{ \pm}=$ $\omega_{\mathrm{m}} \pm \sqrt{\omega_{\mathrm{d}}^{2}+g_{\text {eff }}^{2}}\left[\right.$ see Eq. (2)], where $\omega_{\mathrm{m}}=\left(\omega_{\mathrm{e}}+\omega_{\mathrm{s}}\right) / 2$ and $\omega_{\mathrm{d}}=\left(\omega_{\mathrm{e}}-\omega_{\mathrm{s}}\right) / 2$. The matrix $M$ can be expressed as

$$
M=\omega_{\mathrm{m}}\left(\begin{array}{ll}
1 & 0 \\
0 & 1
\end{array}\right)+\sqrt{\omega_{\mathrm{d}}^{2}+g_{\mathrm{eff}}^{2}}\left(\begin{array}{cc}
\cos \theta & \sin \theta \\
\sin \theta & -\cos \theta
\end{array}\right),
$$

where

$$
\tan \theta=\frac{g_{\text {eff }}}{\omega_{\mathrm{d}}} .
$$

The transformation

$$
\left(\begin{array}{c}
A_{\mathrm{e}} \\
A_{\mathrm{s}}
\end{array}\right)=U\left(\begin{array}{c}
A_{+} \\
A_{-}
\end{array}\right),
$$

where

$$
U=\left(\begin{array}{cc}
\cos \frac{\theta}{2} & -\sin \frac{\theta}{2} \\
\sin \frac{\theta}{2} & \cos \frac{\theta}{2}
\end{array}\right),
$$

which diagonalizes the linear part of $\mathcal{H}_{\mathrm{D}}$, yields

$$
\hbar^{-1} \mathcal{H}_{\mathrm{D}}=\omega_{+} N_{+}+\omega_{-} N_{-}+K_{\mathrm{M}}\left(A_{\mathrm{s}}^{\dagger} A_{\mathrm{s}}\right)^{2},
$$

where $A_{\mathrm{s}}=A_{+} \sin (\theta / 2)+A_{-} \cos (\theta / 2)$, and where $N_{ \pm}=$ $A_{ \pm}^{\dagger} A_{ \pm}$. 
In the rotating wave approximation (RWA) the Hamiltonian (A7) becomes

$$
\begin{aligned}
\hbar^{-1} \mathcal{H}_{\mathrm{D}}= & \omega_{+} N_{+}+\omega_{-} N_{-} \\
& +K_{+} N_{+}^{2}+K_{-} N_{-}^{2}+K_{\mathrm{i}} N_{+} N_{-},
\end{aligned}
$$

where the Kerr coefficients $K_{ \pm}$are given by

$$
\begin{aligned}
& K_{+}=K_{\mathrm{M}} \sin ^{4} \frac{\theta}{2}, \\
& K_{-}=K_{\mathrm{M}} \cos ^{4} \frac{\theta}{2},
\end{aligned}
$$

[1] R. M. Hill and R. S. Bergman, "Nonlinear response of yig," Journal of Applied Physics, vol. 32, no. 3, pp. S227S228, 1961.

[2] R. LeCraw, E. Spencer, and C. Porter, "Ferromagnetic resonance line width in yttrium iron garnet single crystals," Physical Review, vol. 110, no. 6, p. 1311, 1958.

[3] R. Kumar, B. Samantaray, and Z. Hossain, "Ferromagnetic resonance studies of strain tuned bi: Yig films," Journal of Physics: Condensed Matter, vol. 31, no. 43, p. 435802, 2019.

[4] M. Rytel, P. Kopyt, and B. Salski, "Phase locked loop ku band frequency synthesizer based on a tuned yig oscillator," in 2018 22nd International Microwave and Radar Conference (MIKON). IEEE, 2018, pp. 434-437.

[5] C. Tsai, G. Qiu, H. Gao, L. Yang, G. Li, S. Nikitov, and Y. Gulyaev, "Tunable wideband microwave bandstop and band-pass filters using yig/ggg-gaas layer structures," IEEE transactions on magnetics, vol. 41, no. 10, pp. 3568-3570, 2005.

[6] K. Kotzebue and L. Fletcher, "A ferrimagnetically-tuned parametric amplifier," IEEE Transactions on Microwave Theory and Techniques, vol. 13, no. 6, pp. 773-776, 1965.

[7] S. M. Rezende, "Fundamentals of magnonics," Springer, 2020.

[8] S. Demokritov, V. Demidov, O. Dzyapko, G. Melkov, A. Serga, B. Hillebrands, and A. Slavin, "Bose-einstein condensation of quasi-equilibrium magnons at room temperature under pumping," Nature, vol. 443, no. 7110, pp. 430-433, 2006.

[9] X. Zhang, N. Zhu, C.-L. Zou, and H. X. Tang, "Optomagnonic whispering gallery microresonators," Physical review letters, vol. 117, no. 12, p. 123605, 2016.

[10] A. Osada, R. Hisatomi, A. Noguchi, Y. Tabuchi, R. Yamazaki, K. Usami, M. Sadgrove, R. Yalla, M. Nomura, and Y. Nakamura, "Cavity optomagnonics with spinorbit coupled photons," Physical review letters, vol. 116, no. 22, p. 223601, 2016.

[11] D. D. Stancil and A. Prabhakar, Spin waves. Springer, 2009.

[12] Y. Kajiwara, K. Harii, S. Takahashi, J.-i. Ohe, K. Uchida, M. Mizuguchi, H. Umezawa, H. Kawai, K. Ando, K. Takanashi et al., "Transmission of electrical signals by spin-wave interconversion in a magnetic insulator," Nature, vol. 464, no. 7286, pp. 262-266, 2010.

[13] V. Cherepanov, I. Kolokolov, and V. L'vov, "The saga of yig: spectra, thermodynamics, interaction and relaxation and the inter-mode Kerr coefficient $K_{\mathrm{i}}$ is given by $K_{\mathrm{i}}=$ $K_{\mathrm{M}} \sin ^{2} \theta$. of magnons in a complex magnet," Physics reports, vol. 229, no. 3, pp. 81-144, 1993.

[14] A. Serga, A. Chumak, and B. Hillebrands, "Yig magnonics," Journal of Physics D: Applied Physics, vol. 43, no. 26, p. 264002, 2010.

[15] S. Rezende, F. De Aguiar, and O. de Alcantara Bonfim, "Order and chaos in ferromagnetic spin wave instabilities," Journal of Magnetism and Magnetic Materials, vol. 54, pp. 1127-1131, 1986.

[16] S. M. Rezende and F. M. de Aguiar, "Spin-wave instabilities, auto-oscillations, and chaos in yttrium-iron-garnet," Proceedings of the IEEE, vol. 78, no. 6, pp. 893-908, 1990.

[17] S. Sharma, Y. M. Blanter, and G. E. Bauer, "Optical cooling of magnons," Physical review letters, vol. 121, no. 8, p. $087205,2018$.

[18] R. Jepsen, "Harmonic generation and frequency mixing in ferromagnetic insulators," Journal of Applied Physics, vol. 32, no. 12, pp. 2627-2630, 1961.

[19] F. R. Morgenthaler, "Harmonic resonances in small ferrimagnetic ellipsoids," Journal of Applied Physics, vol. 30, no. 4, pp. S157-S159, 1959.

[20] Y.-P. Wang, G.-Q. Zhang, D. Zhang, T.-F. Li, C.-M. Hu, and J. You, "Bistability of cavity magnon polaritons," Physical review letters, vol. 120, no. 5, p. 057202, 2018.

[21] Y.-P. Wang, G.-Q. Zhang, D. Zhang, X.-Q. Luo, W. Xiong, S.-P. Wang, T.-F. Li, C.-M. Hu, and J. You, "Magnon kerr effect in a strongly coupled cavity-magnon system," Physical Review B, vol. 94, no. 22, p. 224410, 2016.

[22] P. Hyde, B. Yao, Y. Gui, G.-Q. Zhang, J. You, and C.-M. $\mathrm{Hu}$, "Direct measurement of foldover in cavity magnonpolariton systems," Physical Review B, vol. 98, no. 17, p. 174423, 2018.

[23] H. Suhl, "The theory of ferromagnetic resonance at high signal powers," Journal of Physics and Chemistry of Solids, vol. 1, no. 4, pp. 209-227, 1957.

[24] G. Wiese and H. Benner, "Multistability and chaos by parametric excitation of longwave modes in a yig sphere," Zeitschrift für Physik B Condensed Matter, vol. 79, no. 1, pp. 119-131, 1990.

[25] M. G. Cottam and Z. Haghshenasfard, "Instability processes for magnons in ferromagnetic nanostructures," Physics in Canada, vol. 72, no. 2, 2016.

[26] M. Elyasi, Y. M. Blanter, and G. E. Bauer, "Resources of nonlinear cavity magnonics for quantum information," arXiv:1910.11130, 2019. 
[27] Z. Zhang, M. O. Scully, and G. S. Agarwal, "Quantum entanglement between two magnon modes via kerr nonlinearity driven far from equilibrium," Physical Review Research, vol. 1, no. 2, p. 023021, 2019.

[28] X. Zhang, C.-L. Zou, L. Jiang, and H. X. Tang, "Strongly coupled magnons and cavity microwave photons," Physical review letters, vol. 113, no. 15, p. 156401, 2014.

[29] Y. Tabuchi, S. Ishino, T. Ishikawa, R. Yamazaki, K. Usami, and Y. Nakamura, "Hybridizing ferromagnetic magnons and microwave photons in the quantum limit," Physical Review Letters, vol. 113, no. 8, p. 083603, 2014.

[30] D. Lachance-Quirion, Y. Tabuchi, A. Gloppe, K. Usami, and Y. Nakamura, "Hybrid quantum systems based on magnonics," Applied Physics Express, vol. 12, no. 7, p. 070101, 2019.

[31] D. Lachance-Quirion, S. P. Wolski, Y. Tabuchi, S. Kono, K. Usami, and Y. Nakamura, "Entanglement-based single-shot detection of a single magnon with a superconducting qubit," arXiv:1910.09096, 2019.

[32] Y. Tabuchi, S. Ishino, A. Noguchi, T. Ishikawa, R. Yamazaki, K. Usami, and Y. Nakamura, "Quantum magnonics: The magnon meets the superconducting qubit," Comptes Rendus Physique, vol. 17, no. 7, pp. 729-739, 2016.

[33] S. V. Kusminskiy, "Cavity optomagnonics," arXiv:1911.11104, 2019.

[34] R. Denton, "Theoretical and experimental characteristics of a ferromagnetic amplifier using longitudinal pumping," Journal of Applied Physics, vol. 32, no. 3, pp. S300-S307, 1961.

[35] X. Zhang, C.-L. Zou, L. Jiang, and H. X. Tang, "Cavity magnomechanics," Science advances, vol. 2, no. 3, p. e1501286, 2016.

[36] J. C. Slonczewski, "Current-driven excitation of magnetic multilayers," Journal of Magnetism and Magnetic Materials, vol. 159, no. 1-2, pp. L1-L7, 1996.

[37] S. I. Kiselev, J. Sankey, I. Krivorotov, N. Emley, R. Schoelkopf, R. Buhrman, and D. Ralph, "Microwave oscillations of a nanomagnet driven by a spin-polarized current," nature, vol. 425, no. 6956, pp. 380-383, 2003.

[38] C. Safranski, I. Barsukov, H. K. Lee, T. Schneider, A. Jara, A. Smith, H. Chang, K. Lenz, J. Lindner, Y. Tserkovnyak et al., "Spin caloritronic nanooscillator," Nature communications, vol. 8, no. 1, pp. 1-7, 2017.

[39] S. Daimon, R. Iguchi, T. Hioki, E. Saitoh, and K.-i. Uchida, "Thermal imaging of spin peltier effect," Nature communications, vol. 7, no. 1, pp. 1-7, 2016.

[40] G. Zhang, Y. Wang, and J. You, "Theory of the magnon kerr effect in cavity magnonics," SCIENCE CHINA Physics, Mechanics \&6 Astronomy, vol. 62, no. 8, p. 987511, 2019.

[41] T. Holstein and H. Primakoff, "Field dependence of the intrinsic domain magnetization of a ferromagnet," Physical Review, vol. 58, no. 12, p. 1098, 1940.

[42] C. Mathai, S. Masis, O. Shtempluck, S. Hacohen-Gourgy, and E. Buks, "Frequency mixing in a ferrimagnetic sphere resonator," Euro. Phys. Lett., vol. 131, 2020.

[43] L. S. de Los Terreros and F. J. Bermejo, "Quantum langevin equations for a two-mode parametric amplifier: Noise squeezing without negative diffusion," Phys. Rev. A, vol. 45, pp. 1906-1918, 1992.

[44] P. Kirton, M. M. Roses, J. Keeling, and E. G. Dalla Torre, "Introduction to the dicke model: From equilibrium to nonequilibrium, and vice versa," Advanced Quantum Technologies, vol. 2, no. 1-2, p. 1800043, 2019.

[45] M. R. Jeffrey, A. R. Champneys, M. di Bernardo, and S. W. Shaw, "Catastrophic sliding bifurcations and onset of oscillations in a superconducting resonator," Phys. Rev. E, vol. 81, no. 1, p. 016213, Jan 2010.

[46] W. Froncisz and J. S. Hyde, "The loop-gap resonator: a new microwave lumped circuit esr sample structure," Journal of Magnetic Resonance (1969), vol. 47, no. 3, pp. 515-521, 1982.

[47] D. Zhang, W. Song, and G. Chai, "Spin-wave magnonpolaritons in a split-ring resonator/single-crystalline yig system," Journal of Physics D: Applied Physics, vol. 50, no. 20, p. 205003, 2017.

[48] J. Krupka, K. Derzakowski, M. Tobar, J. Hartnett, and R. G. Geyer, "Complex permittivity of some ultralow loss dielectric crystals at cryogenic temperatures," Measurement Science and Technology, vol. 10, no. 5, p. 387, 1999.

[49] B. Yurke and E. Buks, "Performance of cavity-parametric amplifiers, employing kerr nonlinearites, in the presence of two-photon loss," J. Lightwave Tech., vol. 24, pp. 5054-5066, 2006.

[50] D. Wood and J. Remeika, "Effect of impurities on the optical properties of yttrium iron garnet," Journal of Applied Physics, vol. 38, no. 3, pp. 1038-1045, 1967.

[51] A. M. Hofmeister, "Thermal diffusivity of garnets at high temperature," Physics and Chemistry of Minerals, vol. 33, no. 1, pp. 45-62, 2006. 